\title{
Seroepidemiología de la enfermedad de Chagas en dos localidades del municipio Costa de Oro, estado Aragua, Venezuela
}

\author{
Olga Serrano1, Florencio Mendoza1', Benny Suárez²,3,4, Ana Soto ${ }^{3,4}$ \\ 1 Corporación de Salud del Estado Aragua, Aragua, Venezuela \\ 2 Postgrado en Epidemiología de Enfermedades Metaxénicas, Aragua, Venezuela \\ ${ }^{3}$ Centro de Investigaciones de Enfermedades Endémicas y Salud Ambiental, Aragua, Venezuela \\ 4 Instituto de Altos Estudios en Salud Pública "Doctor Arnoldo Gabaldon", Aragua, Venezuela
}

Introducción. La enfermedad de Chagas es un problema de salud pública en América Latina. En Venezuela, la prevalencia en zonas marginales de poblaciones rurales y de áreas endémicas para el año 2000 fue de 8,3\%.

Objetivo. Determinar la seroprevalencia de infección por Trypanosoma cruzi en la población menor de 16 años, los indicadores entomológicos y el grado de conocimiento de la enfermedad de Chagas en los habitantes.

Materiales y métodos. Se realizó un trabajo de campo descriptivo de corte transversal en dos comunidades rurales del municipio Costa de Oro, estado Aragua, Venezuela. Se determinó la presencia de anticuerpos para $T$. cruzi en toda la población menor de 16 años mediante las pruebas de hemoaglutinación indirecta y ELISA. Se realizó una encuesta epidemiológica al jefe de familia para la búsqueda de factores de riesgo asociados a la enfermedad.

Resultados. La seroprevalencia en niños menores de 16 años fue de $1,02 \%$. Se recolectaron 16 triatominos de tres especies diferentes: Panstrongylus geniculatus, Rhodnius pictipes y Eratyrus mucronatus. No se encontraron triatominos positivos para T. cruzi. El índice de infestación en el lugar y las casas fue de $100 \%$ y 10,9\%, respectivamente; no se encontró asociación entre la serología positiva para $T$. cruzi y las variables estudiadas. El $95 \%$ de los encuestados conocen el insecto transmisor de la enfermedad, pero menos del $46 \%$ saben qué es la enfermedad, cómo se transmite y los daños que produce.

Conclusión. El hallazgo de vectores secundarios en la zona plantea la necesidad de realizar estudios epidemiológicos y entomológicos que incorporen nuevas variables afines con el hábitat de estos triatominos, de manera que permitan la identificación de los factores de riesgos implicados en la transmisión de esta enfermedad en la zona.

Palabras clave: enfermedad de Chagas/epidemiología, Trypanosoma cruzi, vectores de enfermedades, vigilancia epidemiológica, Venezuela.

Seroepidemiology of Chagas disease in two rural populations in the municipality of Costa de Oro, at Aragua State, northern Venezuela

Introduction. Chagas disease has been a public health problem in Latin America for many years. In Venezuela the disease is mainly distributed in the rural populations and endemic areas with an overall seroprevalence index of $8,3 \%$ for the year 2000 .

Objective. The infection seroprevalence of Trypanosoma cruzi was determined in the human population younger than 16 years. An the entomological index was obtained and the grade of knowledge of the Chagas disease was surveyed in the local inhabitants.

Materials and methods. A descriptive cross-sectional field study was performed in two rural communities of the municipality Costa de Oro, State Aragua, Venezuela. Here, T. cruziseropositive testing was conducted in subjects younger than 16 years. The samples were processed using indirect hemagglutination and by ELISA (enzyme-linked immunosorbent assay). A questionnaire was administered to assess household risk factors, and knowledge 
about vectors and disease held by the local population. Searches for triatomines were conducted inside of dwellings.

Results. Seroprevalence in children less than 16 years old was $1.02 \%$. Sixteen specimens of triatominae adults were captured. The vector species recognized were Panstrongylus geniculatus, Rhodnius pictipes, and Eratyrus mucronatus. The index of triatomid infestation with respect to site and house was respectively, $100 \%$ and $10.9 \%$. No association was found between the seropositives and the other variables. Of the interviewed individuals, 95\% knew that insects transmit diseases, but less than $46 \%$ knew that triatominds transmit Chagas disease or were aware of the disease pathology.

Conclusion. The discovery of secondary vectors in the area outlines the necessity to carry out epidemiological and entomological studies that associate environmental variables with the habitat of these insects. This will allow the identification of risk factors responsible for the transmission of Chagas disease in this region.

Key words: Chagas disease/epidemiology, Trypanosoma cruzi, disease vectors, epidemiologic surveillance, Venezuela.

La Organización Panamericana para la Salud (OPS) describe la enfermedad de Chagas como una patología limitada al continente americano, la cual es endémica, principalmente, en México, América Central y América del Sur (1). Esta enfermedad compromete esencialmente a la población rural de bajo estrato social y está directamente relacionada con la pobreza, la insalubridad y las viviendas de bahareque, con abundantes grietas, piso de tierra, techo de paja y mala iluminación, constituyen un hábitat adecuado para los triatominos vectores (2).

Esta enfermedad se considera un problema de salud pública debido a que disminuye la capacidad productiva de las personas que la padecen. Se estima que, en Venezuela, existen seis millones de personas en riesgo de contraer esta enfermedad, en el país el índice de seroprevalencia para el año 2000 fue de 8,3\% y está distribuida principalmente en la región andina, en el piedemonte y en las montañas de la zona costera $(3,4)$.

Como no existe una terapéutica apropiada ni vacuna para proteger a los individuos, la lucha

\footnotetext{
Correspondencia:

Ana Soto, Centro de Estudios Enfermedades Endémicas y Salud Ambiental, Instituto de Altos Estudios "Doctor Arnoldo Gabaldón”, Avenida Bermúdez Sur № 93, Edificio de Malariología, Maracay, Estado Aragua, Venezuela; apartado postal 2171, 2113/ZIP 2101-A.

Teléfono: (0058) 02432412846

ana.soto@iaesp.edu.ve
}

Recibido: 09/05/07; aceptado: 18/10/07 contra la enfermedad se restringe a la acción contra los vectores, ya sea mediante el uso de insecticidas o la transformación de la vivienda haciéndola no apta para la colonización de los triatominos. La intensa campaña antichagásica realizada en el país desde la década de los 60 , redujo la enfermedad en las zonas consideradas endémicas; la campaña incluyó la lucha contra el vector y el mejoramiento de las viviendas (5). No obstante el Programa de Control de la Enfermedad de Chagas en el país se ha debilitado considerablemente por el resurgimiento de enfermedades como el paludismo y el dengue (6) y por la reducción de recursos por parte del gobierno central para este programa.

El estado Aragua no escapa de esta realidad, lo que ha traído como consecuencia que disminuyan las acciones de control y vigilancia de los triatominos vectores en localidades donde se conoce la presencia de estos insectos. Según información suministrada por la Corporación de Salud del estado Aragua (CORPOSALUD-Aragua) (7), la fase crónica de la enfermedad fue responsable de la muerte de 350 personas en los años 2000 al 2003, con una tasa de 5,65 por 100.000 habitantes. Durante los últimos años ha sido constante la presencia de triatominos en recolecciones realizadas en los municipios Girardot, Tovar, San Casimiro, Camatagua, Urdaneta y Costa de Oro (8). En las comunidades de Cumboto y Periquitos del municipio Costa de Oro, es frecuente la denuncia de los moradores de la presencia de "chipos" en la zona. Según 
datos de CORPOSALUD-Aragua, durante el primer trimestre del 2005 se registró la presencia de Panstrongylus geniculatus (Latreille, 1811) con evidencias de contacto hombre-vector (reacciones alérgicas a picaduras del insecto) y $50 \%$ de infección de triatominos por Trypanosoma cruzi Chagas 1901.

Con la finalidad de contribuir al conocimiento seroepidemiológico de esta enfermedad, se realizó un estudio cuyo objetivo fue determinar la seroprevalencia de infección por $T$. cruzien la población menor de 16 años, conocer los indicadores entomológicos y explorar el grado de conocimiento de la enfermedad de Chagas en los habitantes.

\section{Materiales y métodos}

\section{Área de estudio}

Se realizó un estudio descriptivo de corte transversal de seroprevalencia de $T$. cruzi y su asociación con factores de riesgo, de julio a septiembre de 2005, en las comunidades de Cumboto y Periquito, estado Aragua, Venezuela (figura 1). Este estudio fue aprobado por el Comité de Bioética del Instituto de Altos Estudios en Salud "Doctor Arnoldo Gabaldón".

Las comunidades de Cumboto y Periquito son netamente rurales y están enclavadas en la selva perteneciente al Parque Nacional Henry Pittier, ubicado en una zona boscosa, decidua, semiárida, tropófila, cálida, de tierras bajas, que se desarrolla desde los $200 \mathrm{~m}$ hasta unos $500 \mathrm{~m}$ de altitud. Se ha mencionado como formación vegetal con los nombres de selva veranera (9), bosque montano deciduo (10) y bosque deciduo (11). En el sistema de zonas de vida de Holdridge, queda definido como bosque seco tropical (12). Estas comunidades se encuentran ubicadas a $12 \mathrm{~km}$ y $8 \mathrm{~km}$, respectivamente, de la localidad de Ocumare (capital del municipio,) con la cual se comunican por una carretera pavimentada de dos vías.

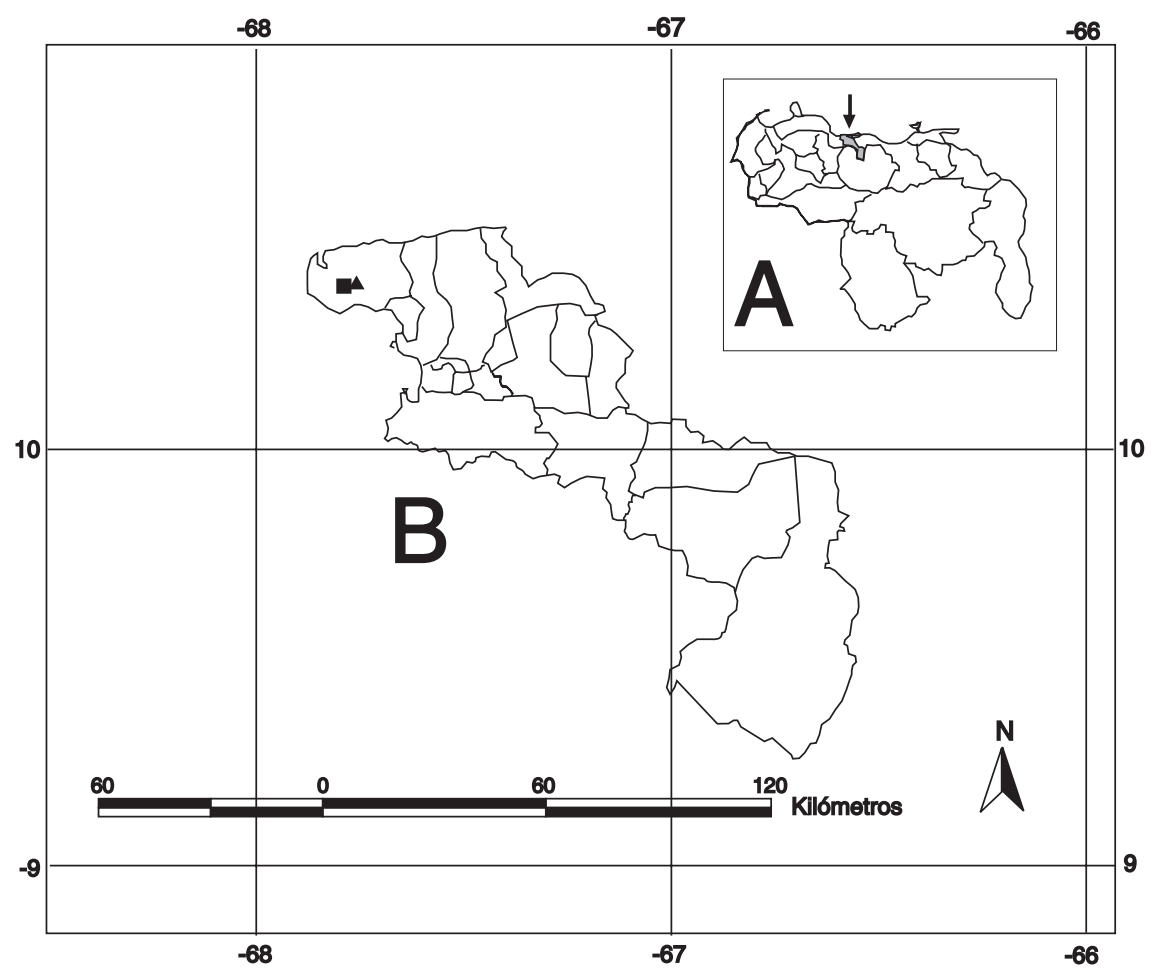

Figura 1. Ubicación geográfica de las localidades de Cumboto y Periquitos. A. Mapa de Venezuela. B. Municipio Costa de Oro. 
Los ríos Cumboto y Trilla corren a lo largo de las comunidades de Cumboto y Periquitos, respectivamente, los cuales confluyen para formar el río Ocumare. La temperatura en la zona varía desde $26^{\circ} \mathrm{C}$ hasta $28^{\circ} \mathrm{C}$. Ambas localidades están provistas de acueducto, servicio eléctrico y calles pavimentadas; su población es joven y de caracter progresivo, con una edad media de 29 años, una tasa de fecundidad de 9,4 y un índice de masculinidad de 53,3 por cada 100 nacimientos femeninos.

Es conveniente mencionar que, antes de la ejecución del trabajo de campo, se informó a los líderes de la comunidad y al personal de salud del área, sobre los objetivos, los alcances y la importancia de su participación en el desarrollo del estudio; la comunidad fue la primera fuente de información para la elaboración del croquis de la zona, la ubicación de las viviendas y la identificación de los hogares donde residían los niños menores de 16 años. La selección de este grupo de edad obedece a la necesidad de conocer si existe transmisión reciente.

\section{Tamaño de muestra y muestreo}

Para estimar la seroprevalencia de infección por T. cruzi, la muestra correspondió al universo de la población menor de 16 años, que cumplió con los criterios de inclusión (autorización y consentimiento por escrito del padre o representante legal del menor, no pertenecer a protocolos de inmunoprofilaxis de leishmaniasis, no haber recibido inmunoterapia para leishmaniasis cutánea). Se realizó una encuesta socioepidemiológica al jefe de familia o representante legal, en las viviendas donde residían los menores, para recoger información referente al grupo familiar, conocimiento de la enfermedad de Chagas, insecto transmisor, características de la vivienda, vegetación alrededor de la casa y presencia de animales dentro y fuera de la vivienda.

\section{Pruebas serológicas}

Se tomaron muestras de sangre en papel filtro, según el protocolo del Laboratorio Nacional de Diagnóstico de Chagas, Ministerio del Poder Popular para la Salud.
Las muestras fueron analizadas en el Laboratorio de Diagnóstico de Chagas del Instituto de Medicina Tropical de la Universidad Central de Venezuela. Éstas se evaluaron mediante la prueba de hemoaglutinación indirecta y el ensayo inmunoenzimático (ELISA). Luego de obtener los resultados de las pruebas, se clasificaron como positivas las que resultaron reactivas en las dos pruebas $(5,13)$. En los casos positivos para $T$. cruzi, se tomaron muestras de sangre venosa a los niños seropositivos y a su madre, para confirmar los resultados y descartar transmisión transplacentaria.

\section{Definición de términos}

Con respecto a las características o el tipo de construcción de las viviendas para este estudio, se definieron casa y rancho. Casa era la vivienda construida con materiales como bloque o ladrillo frisado o sin frisar, concreto, madera aserrada, adobe, techo de concreto, láminas metálicas de zinc en el techo, piso de cemento $u$ otro material que no fuera tierra. Rancho era la vivienda construida con materiales de desechos, tales como tablas, cartón, caña, paredes de zinc o bahareque.

\section{Muestreo entomológico}

Los insectos se recolectaron mediante capturas sistemáticas y no sistemáticas. Para determinar los índices de infestación de los hogares y lugares y el índice de infección triatomínica, se revisaron las viviendas por personal experto por espacio de una hora/hombre, utilizando linternas y pinzas finas. Las capturas no sistemáticas fueron hechas por los habitantes de las viviendas encuestadas durante tres meses, a quienes se les entregó un recipiente con etiqueta y se les entrenó para identificar el rótulo con la fecha, la hora y el lugar de captura de los triatominos (14). Después de la recolección, se identificaron los triatominos empleando la clave de Lent and Wygodzinski (15) y se determinó el grado de infestación e infección por triatominos.

La identificación de la positividad de triatominos para Trypanosoma spp. se hizo por medio de un extendido del contenido intestinal de cada triatomino, el cual fue coloreado por la técnica de 
Giemsa y observado al microscopio con objetivo de inmersión.

\section{Análisis estadístico}

El almacenamiento de los datos se hizo en el programa Excel y se empleó el programa estadístico Epi-Info, versión 6.04. Los factores de riesgos asociados a la transmisión de la enfermedad de Chagas se determinaron utilizando la prueba de ji al cuadrado con corrección de Yates, considerando $p<0,05$ como significativo, con un intervalo de confianza de $95 \%$. Los resultados se presentaron en frecuencias absolutas, porcentajes e índices.

\section{Resultados}

El levantamiento del croquis de la zona indicó que la comunidad cuenta con 376 viviendas, aproximadamente, 200 localizadas en la población de Cumboto $(60 \%)$ y 176 en la población de Periquitos (40\%). Las viviendas donde residían niños menores de 16 años, representó 24\% (92) del total de la zona de estudio.

La seroprevalencia de T.cruzi en la población estudiada fue de 1,02\%; de las 195 muestras, se encontraron dos positivas que correspondían a dos hermanos, uno de sexo masculino con 14 años de edad y una de sexo femenino de 12 años de edad. Estos resultados se confirmaron analizando las muestras de sangre venosa tomada a los positivos.

En relación con las características de las viviendas encuestadas, $20,7 \%$ correspondió a ranchos y, el resto $(79,3 \%)$, a casas. Fueron más frecuentes las casas con paredes de bloque $(78,3 \%)$, frisadas totalmente $(65 \%)$, con techos de zinc $(51,7 \%)$ y piso de cemento $(95 \%)$. En las viviendas catalogadas como ranchos, predominaron las paredes de zinc $(63,2 \%)$, los techos de zinc $(94,7 \%)$ y los pisos de cemento $(68,45 \%)$. El $13 \%$ de todas las viviendas tenían paredes de bahareque.

La vivienda con casos seropositivos para anticuerpos anti- $T$. cruzi, se encuentra ubicada en el caserío Casamacho de la localidad de Periquito, caracterizada por tener paredes de bloque parcialmente frisados, techo de zinc cubierto con asfalto y piso de cemento. No se encontró asociación estadística de la serología positiva a anticuerpos anti- $T$. cruzicon el tipo de vivienda $\left(\mathrm{Ji}^{2}=0,01, \mathrm{p}=0,90\right)$, ni con los materiales de construcción de las paredes $\left(\mathrm{Ji}^{2}=0,30, \mathrm{p}=0,58\right)$, el piso $\left(\mathrm{Ji}^{2}=0,60, \mathrm{P}=0,43\right)$ o el friso $\left(\mathrm{Ji}^{2}=0,81\right.$, $\mathrm{p}=0,36$ ).

El índice de hacinamiento mostró que en $8 \%$ de las viviendas, sus habitantes se encuentran en condiciones de hacinamiento, 32\% parcialmente hacinados y $61 \%$ no hacinados. El índice de hacinamiento en la familia de los seropositivos a la enfermedad de Chagas fue de 22,5. No se evidenció asociación entre los seropositivos y las variables estudiadas: hacinamiento $\left(\mathrm{Ji}^{2}=0,38\right.$, $p=0,53)$, presencia de animales $\left(\mathrm{Ji}^{2}=1,07 \mathrm{p}=0,30\right)$, tipo de anexos $\left(\mathrm{Ji}^{2}=0,09, \mathrm{p}=0,76\right)$, cercanía de la vegetación $\left(\mathrm{Ji}^{2}=0,00, \mathrm{p}=0,97\right)$, ni presencia de vegetación tipo bosque (Fisher $p=0,98$ ).

Se recolectaron seis triatominos adultos en las 92 viviendas examinadas; todos los insectos resultaron negativos para Trypanosoma spp. El índice de infestación de triatominos en las casas de las comunidades de Cumboto y Periquitos fue de $16,3 \%$ y $18,9 \%$, respectivamente. Las especies fueron $P$. geniculatus $(68,8 \%)$, Rhodnius pictipes $(25 \%)$ y Eratyrus mucronatus $(6,3 \%)$. Las especies $P$. geniculatus y $R$. pictipes se encontraron en ambas localidades y, $E$. mucronatus, sólo en Cumboto. En las dos localidades, el índice de infestación de triatominos en las casas fue de 10,9\%: por especie, 9,8\% para $P$. geniculatus, $4,3 \%$ para $R$. pictipes y $1 \%$ para E. mucronatus.

No se recolectaron triatominos en la vivienda donde residían los niños positivos para $T$. cruzi, por lo cual no se encontró asociación entre la presencia de triatominos y la serología positiva para anticuerpos anti $-T$. cruzi $\left(\mathrm{Ji}^{2}=0,80, \mathrm{p}=1,0\right)$.

En relación con los aspectos cognoscitivos sobre el vector, se registró que $89,1 \%$ de los encuestados conoce el insecto y $47,8 \%$ declaró haberlo visto dentro de su domicilio, con mayor frecuencia en la sala $(29,3 \%)$ y el dormitorio $(21,7 \%)$. La comunidad conoce el insecto, pero sólo el $40 \%$ de los encuestados conoce qué es la enfermedad, cómo se transmite (46\%), o los daños 
que produce (41\%). Sin embargo, el $85 \%$ considera que la presencia de este insecto es un problema en su comunidad.

\section{Discusión}

El Programa de Control de la Enfermedad de Chagas cambió significativamente los indicadores epidemiológicos de esta nfermedad en el país en las últimas décadas. Según el reporte de la OMS (3), la prevalencia de anticuerpos anti- T. cruzipara Venezuela es de 8,3\%. Persisten focos importantes en algunos estados, como Barinas, Apure, Portuguesa y Cojedes, y, entre 1996 y 1999, cifras de prevalencia que muestran que los niveles de infección en niños menores de 10 años permanecen por encima del objetivo de $0,5 \%$ del programa (5), como 1,3\% en Portuguesa, 0,9\% en Barinas y $0,8 \%$ en Yaracuy. Para el año 2004, en el estado Aragua (aun cuando no se han realizado estudios de seroprevalencia de la enfermedad), de 737 muestras procesadas ocho resultaron positivas, para una seropositividad de 1,08\% (8).

Los resultados de este estudio reportan una seropositividad en niños menores de 16 años de $1,02 \%$ para las localidades en estudio, registrando un porcentaje parecido al reportado para el estado Yaracuy en niños de 5 a 10 años de 1,6\% y jóvenes de 11 a 15 años de 2,2\% (16); la seroprevalencia en menores de 10 años reportada por la OMS para el año 2002 fue de 1\% para Venezuela, con datos de los años 1996 a 1999.

La prevalencia encontrada en este estudio se encuentra por encima del $0,28 \%$ registrada en el país por el Programa de Control de la Enfermedad de Chagas (17), en grupos de edad similares durante el último quinquenio (2000-2004).

Aun cuando no se encontró asociación entre los seropositivos y la presencia de triatominos en la vivienda, la transmisión vectorial no puede descartarse, ya que los familiares refieren la presencia de los triatominos en su casa, inclusive dentro de las habitaciones, los niños siempre han vivido en esta comunidad y no tienen antecedentes de haber viajado a zonas chagásicas, no refieren transfusiones de sangre y la madre resultó negativa para anticuerpos anti- $T$. cruzi. Todo esto descarta la posibilidad de una infección adquirida en otra zona, por transfusión de sangre o transplacentaria y la transmisión vectorial queda como la causa probable de infección.

Por otra parte, no se encontró asociación entre los seropositivos y el resto de los factores de riesgo estudiados, lo cual coincide con otras publicaciones (18), en las cuales no se encontró relación entre el tipo de vivienda y la presencia de triatominos. No obstante, no coincide con reportes (16) que señalan la asociación entre el tipo de viviendas y la seropositividad.

La OMS (3) refiere que los indicadores entomológicos han venido en ascenso de un índice de casa de $0,7 \%$, en 1990 , a $5,2 \%$, en el año 2000. El principal vector de la enfermedad de Chagas en Venezuela es $R$. prolixus. El alto porcentaje de casas construidas con techos de palma y paredes de bahareque en el área rural permitió que este insecto se adaptara fácilmente a vivir dentro de este tipo de casas. La colonización de estas viviendas por $R$. prolixus dio origen a la definición de áreas de riesgo y las estrategias de control se basaron en las características biológicas y el comportamiento de este insecto. En Cumboto y Periquitos son pocos los ranchos y predominan las casas; esto podría explicar la ausencia de $R$. prolixus en la zona durante el estudio. En este estudio sólo se recolectaron vectores secundarios, como $P$. geniculatus, $R$. pictipes y $E$. mucronatus que, por ser triatominos, se consideran potencialmente vectores. Sin embargo, los factores del comportamiento de cada uno de estas especies pueden determinar su capacidad vectorial.

El hecho de no haber encontrado $R$. prolixus en ninguna de las viviendas sugiere que los vectores secundarios hallados vienen ganando importancia en la medida en que han avanzado los programas de control (aplicación de insecticidas o mejoramiento de la vivienda), ya que, con la eliminación del vector primario, se ha aumentado el riesgo de su reemplazo por los vectores secundarios autóctonos de la zona.

En el país se ha reportado $P$. geniculatus en el interior de las casas, en estados como Miranda, Distrito Federal y Aragua (Cuyagua). Según 
algunos reportes, este insecto puede introducirse y multiplicarse en cualquier tipo de vivienda, independientemente del tipo y calidad de la construcción $(19,20)$. De igual manera, se ha reportado infestación mixta de $R$. prolixus y $P$. geniculatus en una zona endémica del estado Lara, por lo cual los autores sugieren la posible domiciliación y competencia con $R$. prolixus por el nicho ecológico (6). Según algunos reportes, en el estado Yaracuy la población en general identifica a $P$. geniculatus como la especie observada con mayor frecuencia. Este insecto se ha encontrado con infección natural por T. cruzi, y positividad a sangre mixta humana y animales domésticos, como perros, conejos y cochinos, lo que indica un contacto hombre-insecto $(20,21)$. En el caso de E. mucronatus, se ha reportado en el domicilio (22) en el estado Trujillo; en ese reporte se sugiere la necesidad de estudiar la posibilidad de proceso de domiciliación del vector.

Nuestros resultados indican la necesidad de buscar nuevas estrategias para el estudio de estos vectores, ya que rutinariamente se realizan inspecciones entomológicas en busca de insectos transmisores de la enfermedad de Chagas y se utilizan los métodos clásicos para la búsqueda de $R$. prolixus en las viviendas, por esta razón, se pueden pasar por alto las viviendas que no posean estas características, al considerar que allí es poco probable que se encuentren insectos vectores, y se obvian variables que indiquen otros factores de riesgo ligados a la presencia de vectores secundarios que pudieran estarse adaptándose a hábitats peridomiciliarios y domésticos y que pueden convertirse en vectores importantes de la enfermedad en ciertas áreas bien localizadas.

En este sentido, se sugiere diseñar un sistema de vigilancia entomológico que incorpore variables que permitan la búsqueda de factores de riesgo para la transmisión de la enfermedad, con base en aspectos bioecológicos de los vectores secundarios autóctonos de la zona. También, se sugiere ampliar el criterio de áreas de riesgo para la enfermedad, de manera que no se limite sólo a comunidades que presenten características relacionadas con el hábitat de $R$. prolixus, sino que se incorporen variables que puedan asociarse con los vectores secundarios, los cuales se reportan frecuentemente en el estado.

Con base en los resultados obtenidos en las encuestas sobre los aspectos cognoscitivos de la enfermedad, en este estudio se pudo constatar, al igual que en el estado Barinas, que las personas "conocen" el vector porque lo han visto más dentro y alrededor de las viviendas, pero no lo relacionan con la enfermedad ni la conocen. Es diferente en el estado Portuguesa, donde las personas reconocen menos al "chipo" (probablemente porque ha sido más controlado), saben más sobre la enfermedad y han conocido más personas que la han padecido (23).

Nuestro hallazgo podría explicarse por la poca actividad educativa en las comunidades sobre la enfermedad y su vector, lo que sugiere que la reducción progresiva de los recursos para el Programa de Control de la Enfermedad de Chagas por parte del gobierno central en los últimos años, ha traído como consecuencia el desconocimiento de esta enfermedad.

Los aportes de este estudio son básicos para el conocimiento de la epidemiología de la enfermedad de Chagas en zonas rurales del estado Aragua. Se requieren estudios sobre la bioecología de los triatominos presentes en la zona, con el fin de contribuir al establecimiento de un sistema de vigilancia entomológica e implementar medidas de prevención acordes con la realidad de la zona.

\section{Agradecimientos}

A las comunidades de Cumboto y Periquito por su valiosa colaboración y apoyo durante el trabajo de campo. El primer autor agradece a la Corporación de Salud del estado Aragua por el apoyo durante la ejecución de la investigación.

\section{Conflicto de intereses}

Los autores manifiestan que los resultados obtenidos en esta investigación no están relacionados con ningún tipo de intereses.

\section{Financiación}

Este estudio fue financiado por la Corporación de Salud del Estado Aragua. 


\section{Referencias}

1. Organización Panamericana de la Salud. Enfermedad de Chagas. Boletín Epidemiológico. 2003; 24:3.

2. Briceño LR. La casa enferma. Caracas: Fondo Editorial Acta Científica Venezolana; 1990. p.50.

3. Organización Mundial de la Salud. Análisis preliminar de la situación de salud en Venezuela. Caracas: Publicación Científica Venezolana; 2002.

4. Ache A, Matos A. Interrupting Chagas disease transmision in Venezuela. Rev Inst Med Trop S Paulo. 2001;43:37-43.

5. Añez AM, Crisante G, Rojas A, Díaz N, Anez N, Carrasco $\mathrm{H}$, et al. La cara oculta de la enfermedad de Chagas en Venezuela. Bol Malariol Salud Ambient. 2003;43:45-57.

6. Feliciangeli MD, Campbell-Lendrum D, Martínez C, González D, Coleman P, Davies C. Chagas disease control in Venezuela: Lessons from the Andean region and beyond. Trends Parasitol. 2003;19:44-9.

7. Corporación de Salud del Estado Aragua. Análisis de situación de salud del Estado Aragua año 2003. Aragua: Corporación de Salud del Estado Aragua; 2003.

8. Corporación de Salud del Estado Aragua. Informe del Programa de Control de Enfermedades Endémicas del Estado Aragua año 2004. Aragua: Corporación de Salud del Estado Aragua; 2004.

9. Schafer E, Phelps WH. Las aves del Parque Nacional Henri Pittier (Rancho Grande) y sus funciones ecológicas. Boletín de la Sociedad Venezolana de Ciencias Naturales. Caracas. 1954;83:1-167.

10. Montaldo P. Principios ecológicos en la determinación de unidades básicas y su aplicación para el estado Aragua, Venezuela. Revista de la Facultad de Agronomía (Maracay). 1966;10:5-87.

11. Fernández B, Ulloa G. Fauna del Parque Henri Pittier. Venezuela. Composición y diversidad de la mastofauna. Acta Científica Venezolana. 1990;91:50-63.

12. Ewel J, Madriz. Zonas de vida de Venezuela. Memoria explicativa sobre el mapa ecológico. Ministerio de Agricultura y Cría, Dirección de Investigación, Ediciones de Fondo Nacional de Investigaciones Agropecuarias. Caracas: Editorial Sucre; 1968. p.264.

13. Organización Mundial de la Salud. Important progress in the control of Chagas disease in South America. Geneve: WHO; 1997.
14. Wolff M, Castillo D. Evidencias de domesticación y aspectos biológicos de Panstrongylus geniculatus (Latreille, 1811) (Hemiptera Reduviidae). Acta Entomol Chil. $2001 ; 24: 77-83$.

15. Lent $\mathbf{H}$, Wygodzinsky $\mathbf{P}$. Revision of the Triatominae (Hemiptera, Reduviidae) and their significance as vectors of Chagas' disease. Bull Amer Mus Nat Hist. 1979;163:123-520.

16. Castillo S, Álvarez C, Bofante C, Gil A, Bofante R, Loyo Y, et al. Seroprevalencia de Trypanosoma cruzi y factores de riesgo en comunidades rurales del municipio Nirgua estado Yaracuy 2003. Boletín Médico de Postgrado de la UCLA. 2004;20:77-9.

17. Ministerio de Salud y Desarrollo Social. Informe del programa control de la Enfermedad de Chagas en Venezuela Año 2004. Caracas: Ministerio de Salud y Desarrollo Social; 2004.

18. Cannova D. Seroepidemiología de tripanosomiasis americana, sector Las Cuevas Estado Carabobo. Revista de la Facultad de Ciencias de la Salud (Universidad de Carabobo). 2003;7:28-33.

19. Reyes-Lugo M, Rodríguez-Acosta A. Domiciliation of the sylvatic Chagas disease vector Panstrongylus geniculatus Latreille, 1811, Triatominae: Reduviidae) in Venezuela. Trans R Soc Trop Med Hyg. 2000;94:508.

20. Carrasco H, Torrellas A, García C, Segovia M, Feliciangeli D. Risk of Trypanosoma cruzi I (Kinetoplastida: Trypanosomatidae) transmisión by Panstrongylus geniculatus (Hemiptera: Reduviidae) in Caracas (Metropolitan District) and neighboring status, Venezuela. Inter J Parasitol. 2005;35:1379-84.

21. Feliciangeli $\mathbf{M}$, Carrasco $\mathbf{H}$, Patterson J, Suárez B, Martínez C, Medina M. Mixed domestic infestation by Rhodnius prolixus and Panstrongylus geniculatus Latreille, 1811, vector incrimination, and seroprevalence for Trypanosoma cruzi among inhabitants in El Guamito, Lara State, Venezuela. Am J Trop Med Hyg. 2004;71:501-5.

22. Soto A, Barazarte $H$, Molina de Fernández D. Primer registro de Eratyrus mucronatus Stal, 1859. (Hemiptera: Reduviidae) en el ambiente domiciliario en Venezuela. Entomotropica 2001;16:215-7.

23. Suárez B, Hernández M, Duque N, Martínez C, Feliciangeli D. Conocimientos sobre la enfermedad de Chagas en los estados Barinas y Portuguesa, Venezuela. Bol Malariol Salud Ambient. 2004;44:109-18. 